\title{
Robust Residual Selection for Fault Detection
}

\author{
Hamed Khorasgani*, Daniel E. Jung**, Gautam Biswas*, Erik Frisk**, and Mattias Krysander**
}

\begin{abstract}
A number of residual generation methods have been developed for robust model-based fault detection and isolation (FDI). There have also been a number of offline (i.e., design-time) methods that focus on optimizing FDI performance (e.g., trading off detection performance versus cost). However, design-time algorithms are not tuned to optimize performance for different operating regions of system behavior. To do this, would need to define online measures of sensitivity and robustness, and use them to select the best residual set online as system behavior transitions between operating regions. In this paper we develop a quantitative measure of residual performance, called the detectability ratio that applies to additive and multiplicative uncertainties when determining the best residual set in different operating regions. We discuss this methodology and demonstrate its effectiveness using a case study.
\end{abstract}

\section{INTRODUCTION}

Model based diagnosis methods use analytical redundancy in the system model for fault detection and isolation. Analytic residual generation and analysis methods are typically used for fault detection, and analysis of non zero residuals forms the basis for fault isolation in the system [1]. However, the robustness and accuracy of residual detection methods can be affected by uncertainties, such as parameter uncertainty, structural uncertainty, measurement noise, and disturbances generated by the environment that affect system behavior. Structural uncertainty is attributed to inaccuracies in the system model because of the lack of complete knowledge of the real processes that make up the system, or approximations that may be made to simplify the computational aspects of the model. Parameter uncertainties arise because of practical limitations in making a sufficient number of measurements to accurately estimate the parameters, and the fact that system parameters may vary with operating conditions that are hard to quantify mathematically. Other sources of uncertainties in system models and behavior prediction can be attributed to computational issues, such as the use of finite difference methods as an approximation for solving partial differential equations, and the need to find approximate solutions for stiff systems to avoid computational complexity in online analysis [2], [3].

It is possible to reduce uncertainties in residual analysis by developing more accurate and complex models but there are no practical solutions to eliminating uncertainty in the analyses. Therefore, uncertainties must be accounted for in the design of any supervisory system. There are two general approaches to deal with uncertainty in supervisory systems. The first approach, active fault detection, identifies uncertainties and applies an auxiliary input to decouple them

\footnotetext{
*Inst. of Software Integrated Systems, Vanderbilt University, USA, Email: \{hamed.g.khorasgani, gautam.biswas\} @vanderbilt.edu **Dept. of Electrical Engineering, Linköping University, Sweden, Email: \{daner, frisk, matkr\}aisy.liu.se
}

from the residuals. These approaches use different methods such as Bayesian filters, optimization methods, geometric control approaches, and robust control methods to perform partial or complete decoupling. They work well if there are few uncertainties in the system (see, for example, [1], [4], [5] and [6]). The second method, passive fault detection, develops robust decision making methods that utilize an adaptive threshold to achieve robustness [7], [8]. Performance measures that tradeoff accuracy versus false alarm rates are often used to derive the threshold values. Passive and active methods used for robust fault detection are reviewed in [9]. In relation to previous work, the methodology described in this paper quantifies a performance measure for each residual in the presence of uncertainties. Online analytic optimization methods may then be employed to select the most robust set of residuals for each operating region.

A fault $f$, is detectable if there is at least one residual that is sensitive to this fault. However, in most systems where a sufficient number of measurements can be made, there are usually multiple residual candidates that are sensitive to the fault. For a model with no uncertainties, all residuals linked to a fault will perform equally well, since deviations from zero can only be attributed to faults. However, when uncertainties are present, a non zero residual may be attributed to fault and/or uncertainty effects. Moreover, the sensitivity of residuals to faults and uncertainty effects can vary in different operating regions of system behavior. Therefore, it is possible that one residual has better detectability performance for a fault in one operating region, but another residual has better performance in another region of operation. Ideally, a FDI system should switch between residuals to optimize detectability performance over multiple operating regions.

Djeziri, et al. [7] present a method for handling parameter uncertainties in the model by deriving adaptive thresholds to avoid false alarms. The uncertainties in the parameters affect the prediction of system dynamics, and this is accounted for in the residuals. Therefore, the approach quantifies the diagnosability performance of the different residuals.

For time-discrete descriptor models with additive faults and noise, Eriksson, et al. [10] propose a diagnosability measure which can be used for quantifying diagnosability performance. This measure is used for online selection of the best set of residuals for different conditions [11]. For timecontinuous dynamic systems with multiplicative faults and uncertainties, the diagnosability performance varies over time and depends on the operating regions and system dynamics. With respect to previous work, a contribution here is a measure for quantifying the performance of residuals in different operating regions by using sensitivity analysis [12].

Our goal in this paper is to develop a quantitative measure of diagnosability performance for residuals in the presence of 
faults and uncertainties. By using a quantitative measure of performance that takes into account sensitivity to faults over that of uncertainties, appropriate residuals can be selected for different operating regions of system behavior. This can be used for offline analyses [13], but the focus in this paper is on online test selection for fault detection across different operating regions of system behavior.

The outline of this paper is as follows. In Section II, we present a formal definition of the problem, and develop a running example to illustrate the methodology that we develop. Section III reviews the theoretical background of the work and Section IV introduces the quantitative measure for the residual performance, called the detectability ratio. The applicability of the method is presented in Section V through a case study. Section VI concludes the paper and discusses future work.

\section{PRoblem Formulation}

\section{A. Model of the system}

In this paper, we restrict our analyses to linear timeinvariant dynamic systems. Though the system parameters for such a model are constant, their values may not be accurately determinable because of lack of complete knowledge about the system and the inability to make sufficient measurements to estimate the parameters. Their values may also be a function of the operating conditions, and change as the system degrades. The state space representation of such a system model may be described as:

$$
\begin{aligned}
\dot{x} & =A(f, \delta) x+B_{u}(f, \delta) u+b_{f}(f, \delta) \\
y & =C(f, \delta) x+d_{f}(f, \delta),
\end{aligned}
$$

where $x$ is the state vector, $u$ is the input vector, $y$ is the output vector, $f$ represents the vector of possible faults, and $\delta$ represents the uncertainty and disturbance vector in the system. As shown in Fig. 1, parameter uncertainties have multiplicative effects on system behavior, whereas disturbances have additive effects. Faults can have additive (e.g., sensor and actuator faults) and multiplicative (parameter faults) effects. Each component parameter in the system may

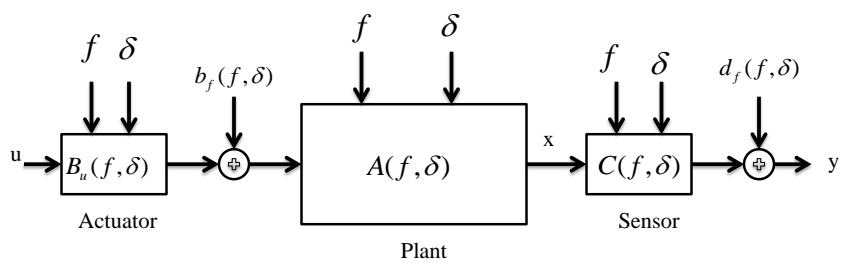

Fig. 1. Linear dynamic system with multiplicative and additive faults and uncertainties.

vary from its nominally specified value due to uncertainties and fault occurrences. This can be represented as: $C_{n}(1+$ $\left.\delta_{n}\right)\left(1+f_{n}\right)$, where $C_{n}$ is the presumed nominal value of the component parameter, and $\delta_{n}$ and $f_{n}$ represent uncertainties and faults associated with the component. It is also assumed that each uncertainty $\delta_{n}$ magnitude has a known upper bound $\Delta_{n}$ and each fault $f_{n}$ magnitude has a lower bound $F_{n}$ :

$$
\left|\delta_{n}\right| \leq \Delta_{n} \quad\left|f_{n}\right| \geq F_{n} .
$$

\section{B. Running example}

As a running example for this paper, consider the following dynamic system

$$
\begin{array}{ll}
\dot{x}_{1}=-\left(1+\delta_{1}\right) x_{1}+u_{1}+f & y_{1}=\left(1+\delta_{3}\right) x_{1} \\
\dot{x}_{2}=x_{1}-2\left(1+\delta_{2}\right) x_{2}+u_{2} & y_{2}=\left(1+\delta_{4}\right) x_{2},
\end{array}
$$

where $x_{1}$ and $x_{2}$ are the system state variables, $u_{1}$ and $u_{2}$ represent the inputs to the system, $y_{1}$ and $y_{2}$ are the system measurements, $\delta_{1}$ and $\delta_{2}$ represent system uncertainties, $\delta_{3}$ and $\delta_{4}$ represent model uncertainties in the sensors, and $f$ is an actuator fault. Fig. 2 illustrates the system model in block diagram form.

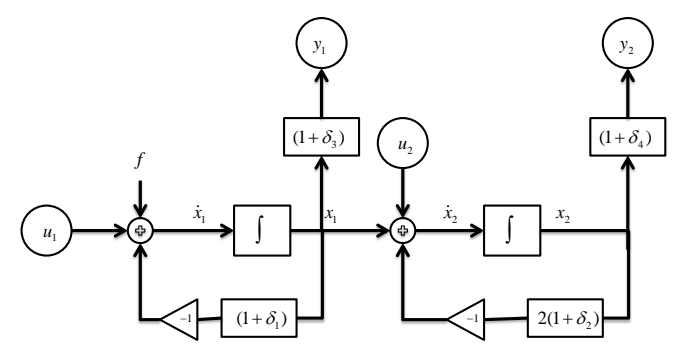

Fig. 2. Simple example diagram.

\section{BACKGROUND}

In this section, we briefly present the model-based diagnosis and sensitivity analysis approaches.

\section{A. Model-based Fault Detection}

A residual (also called an Analytic Redundancy Relation $(A R R))$ is a function of a subset of observables of the system ( $y$ and $u$ in equation (1)). Under nominal conditions it has a value 0 , but may report non zero values in the presence of faults, noise and uncertainties in the system [1]. In the example, $u_{1}, u_{2}, y_{1}$ and $y_{2}$ represent the observed variables from which sets of residuals can be created. These variables are presented as circles in Fig. 2.

A possible residual to detect fault $f$, can be derived from the relation between $u_{1}$ and $y_{1}$ (see equation (3)). Using a derivative causality formulation, the residual can be expressed as:

$$
r_{1}=\dot{y_{1}}+y_{1}-u_{1} \text {. }
$$

Another residual sensitive to fault $f$, can be derived from the relation between $u_{1}, u_{2}$, and $y_{2}$ :

$$
r_{2}=\ddot{y_{2}}+3 \dot{y_{2}}+2 y_{2}-u_{1}-u_{2}-\dot{u}_{2} .
$$

When uncertainty is not a factor, both $r_{1}$ or $r_{2}$ are equally effective in detecting $f$ since any deviation from zero can only be explained by the fault. The only difference in this case would be the rise time of each residual, i.e., how fast they react to the fault. But when there is uncertainty in the system, the performance of the two residuals may differ significantly. Consider the simple example when $\delta_{3} \neq 0$, and $\delta_{1}=\delta_{2}=\delta_{4}=0$. In this case, $r_{2}$ is preferred because it does not use the first measurement $y_{1}$, which is affected by the uncertainty $\delta_{3}$. On the other hand, if $\delta_{1}=\delta_{2}=\delta_{3}=0$ and $\delta_{4} \neq 0$, then $r_{1}$ is the better choice, since $r_{1}$ does not depend 
on $y_{2}$, which is affected by $\delta_{4}$. Thus, the performance of a residual depends on how sensitive it is to a fault as compared to uncertainties that also affect the residual. We build on this simplistic example to develop a sensitivity analysis method for evaluating residuals.

\section{B. Sensitivity Analysis of ODEs}

Sensitivity analysis [12] provides the basis for evaluating the effect of faults and uncertainties on residuals of dynamic systems. Consider a general dynamic system model of the form:

$$
g(\dot{x}, x, u, \theta)=0 \quad h(y, x, \theta)=0,
$$

where $x \in \mathbb{R}^{l_{x}}$ are state variables, $u \in \mathbb{R}^{l_{u}}$ are actuator signals, $y \in \mathbb{R}^{l_{y}}$ are measurement signals, and $\theta \in \mathbb{R}^{l_{\theta}}$ are model parameters.

Sensitivity analysis helps to evaluate how model behaviors are affected by variations in model parameters $\theta$ [14]. When performing sensitivity analysis, the derivative of (6) with respect to each parameter $\theta_{k}$ is computed as:

$$
\begin{array}{r}
\frac{\partial g}{\partial \dot{x}} \dot{p}_{\theta_{k}}+\frac{\partial g}{\partial x} p_{\theta_{k}}+\frac{\partial g}{\partial u} \underbrace{\frac{\partial u}{\partial \theta_{k}}}_{=0}+\frac{\partial g}{\partial \theta_{k}}=0 \\
\frac{\partial h}{\partial y} q_{\theta_{k}}+\frac{\partial h}{\partial x} p_{\theta_{k}}+\frac{\partial h}{\partial \theta_{k}}=0,
\end{array}
$$

where $p_{\theta_{k}}=\frac{\partial x}{\partial \theta_{k}}$ and $q_{\theta_{k}}=\frac{\partial y}{\partial \theta_{k}}$ represent the sensitivity of state and measurement variables to a deviation in parameter $\theta_{k} \cdot \dot{p}_{\theta_{k}}=\frac{\partial \dot{x}}{\partial \theta_{k}}$ and it is assumed that $u$ is an external control signal which does not depend on $\theta_{k}$, implying $\frac{\partial u}{\partial \theta_{k}}=0$.

\section{QUANTITATIVE ANALYSIS OF RESIDUAL DETECTABILITY PERFORMANCE}

In this section, we define a quantitative diagnosability measure using the sensitivity analysis formulation. A small example is used to show how to evaluate the performance of different residuals.

\section{A. Detectability Analysis}

A residual, $r(y, u)=0$, captures nominal system behavior. Ideally, each residual should be zero in the fault-free case and residuals sensitive to a fault become nonzero when it occurs. Due to uncertainties and noise, the residual may deviate from zero even in the fault-free case, and this complicates the fault detection task. To design the fault detection scheme to have a low false alarm rate, a threshold must be derived that separates the uncertainties from the faults. Depending on the relationship between fault and uncertainty magnitudes, the likelihood of detecting a fault of a specified magnitude can vary. If we can quantify this relation, we can use it to select the best residual for detecting individual faults.

A common approach for evaluating detectability performance is to compare the ratio between the signal to be selected and the disturbance, for example the signal-to-noise ratio (SNR). In this case the key factor is the fault magnitude to noise ratio [10]. Also when designing robust residual generators using the $H_{\infty}$ norm, the ratio between faults and disturbances is optimized [15]. To achieve good fault detectability performance with a low risk of false alarms, the ratio of the fault magnitude to parameter uncertainties should be large. A larger ratio corresponds to a fault that is easier to detect since its impact relative to uncertainties will be larger.

\section{B. Detectability Ratio}

Since the effect of uncertainties and faults in the residuals are unknown, we estimate them using sensitivity analysis. It is assumed that the fault magnitude and uncertainties are small. So a first order linear approximation of the effect on a residual with respect to $f$ and $\delta$ is justified, and given by:

$$
r(y, u) \approx \frac{\partial r}{\partial f} f+\sum_{i=1}^{N} \frac{\partial r}{\partial \delta_{i}} \delta_{i}
$$

where the partial derivatives are given by:

$$
\frac{\partial r}{\partial f}=\frac{\partial r}{\partial y} \frac{\partial y}{\partial f}=\frac{\partial r}{\partial y} q_{f}, \quad \frac{\partial r}{\partial \delta_{i}}=\frac{\partial r}{\partial y} \frac{\partial y}{\partial \delta_{i}}=\frac{\partial r}{\partial y} q_{\delta_{i}} .
$$

The parameters $q_{f}$ and $q_{\delta_{i}}$ represent the sensitivity of the sensor signals to the fault and the uncertainties, respectively. Faults and uncertainties are assumed to not affect the input $u$. Note that the term $\frac{\partial r}{\partial y}$ is not a function of fault and uncertainties, and the effects of faults and uncertainties on the residuals are through the sensor signals.

Since the actual magnitudes of the fault and uncertainties are unknown, the maximum values of the magnitude of uncertainties and minimum magnitudes of the fault are used to calculate the worst case scenario of the difficulty in detecting a fault. A quantitative measure of detectability performance is defined as follows.

Definition 1: (Detectability Ratio) Given the dynamic system in equation (1) the detectability ratio of fault $f$ for residual $r$ in the presence of uncertainties $\delta_{i}: i=1,2, \ldots N$ is defined as:

$$
D(f \mid r)=\frac{\left|\frac{\partial r}{\partial f} F_{\min }\right|}{\left|\frac{\partial r}{\partial f} F_{\min }\right|+\sum_{i=1}^{N}\left|\frac{\partial r}{\partial \delta_{i}} \Delta_{i}\right|},
$$

where $\Delta_{i}$ is the absolute value of the upper bound on the magnitude of uncertainty $\delta_{i}$ and $F_{\min }$ is lower bound on the magnitude of fault, $f$. If $\left|\frac{\partial r}{\partial f} F_{\min }\right|=0$, then $D(f \mid r)=0$.

The detectability ratio lies in the interval $[0,1]$ where 0 corresponds to situation that the fault will not affect the residual. The value 1 implies there are no uncertainties affecting the residual. If the effect of a fault is larger than the total effects of the uncertainties, i.e., $D(f \mid r)>0.5$, which means that the fault can be reliably detectable without error. Note that the detectability ratio is not constant but depends on the state and operating region of the system. Higher detectability ratios imply lower thresholds can be chosen to avoid missing alarms, and fault detection time can be reduced.

1) System sensitivity to uncertainties and fault: Consider the system (1) with $N$ uncertainties $\delta_{i}: i=1,2, . ., N$ and a possible fault $f$ in the system. For each of the uncertainties $\delta_{i}$ we define $p_{\delta i}=\frac{\partial x}{\partial \delta_{i}}$ and $q_{\delta i}=\frac{\partial y}{\partial \delta_{i}}$. Also for fault $f$ we have $p_{f}=\frac{\partial x}{\partial f}$ and $q_{f}=\frac{\partial y}{\partial f}$. Therefore, 


$$
\begin{array}{r}
\dot{p_{\delta i}=A p_{\delta i}}+\frac{\partial A}{\partial \delta_{i}} x+\frac{\partial B_{u}}{\partial \delta_{i}} u+\frac{\partial B_{f}}{\partial \delta_{i}} \\
q_{\delta i}=C p_{\delta i}+\frac{\partial C}{\partial \delta_{i}} x+\frac{\partial D_{f}}{\partial \delta_{i}}, \\
\dot{p_{f}=A p_{f}}+\frac{\partial A}{\partial f} x+\frac{\partial B}{\partial f} u+\frac{\partial B_{f}}{\partial f} \\
q_{f}=C p_{f}+\frac{\partial C}{\partial f} x+\frac{\partial D_{f}}{\partial f} .
\end{array}
$$

Note that to perform sensitivity analysis for the system in equation (1), partial derivatives of each of the matrices $A(f, \delta), B_{u}(f, \delta), C(f, \delta)$ and vectors $b_{f}(f, \delta)$ and $d_{f}(f, \delta)$ with respect to fault $f$ and uncertainty $\delta$ must be computable and bounded. Moreover, since the state variables $x$ appeared in equation (10) and equation (11) the linear system represented in equation (1) should be stable and observable.

As an example we compute $p_{\delta 1}=\frac{\partial x}{\partial \delta_{1}}, q_{\delta 1}=\frac{\partial y}{\partial \delta_{1}}, p_{\delta 2}=$ $\frac{\partial x}{\partial \delta_{2}}$ and $q_{\delta 2}=\frac{\partial y}{\partial \delta_{2}}$ for the running example in equation (3) as:

$$
\begin{array}{ll}
\dot{p}_{\delta 1_{1}}=-p_{\delta 11}-x_{1} & \dot{p}_{\delta 2_{1}}=-p_{\delta 21} \\
\dot{p}_{\delta 1_{2}}=p_{\delta 11}-2 p_{\delta 12} & \dot{p}_{\delta 2_{2}}=p_{\delta 21}-2 p_{\delta 22}-2 x_{2} \\
q_{\delta 11}=p_{\delta 11} & q_{\delta 21}=p_{\delta 21} \\
q_{\delta 1_{2}}=p_{\delta 12} & q_{\delta 22}=p_{\delta 22} .
\end{array}
$$

For sensor uncertainties $\delta_{3}$ and $\delta_{4}$ we have:

$$
\begin{array}{ll}
\dot{p}_{\delta 3_{1}}=-p_{\delta 31} & \dot{p}_{\delta 4_{1}}=-p_{\delta 41} \\
\dot{p}_{\delta 3_{2}}=p_{\delta 3_{1}}-2 p_{\delta 3_{2}} & \dot{p}_{\delta 4_{2}}=p_{\delta 4_{1}}-2 p_{\delta 2_{2}} \\
q_{\delta 31}=p_{\delta 31}+x_{1} & q_{\delta 4_{1}}=p_{\delta 41} \\
q_{\delta 32}=p_{\delta 32} & q_{\delta 42}=p_{\delta 4_{2}}+x_{2},
\end{array}
$$

where $p_{\delta 3}=\frac{\partial x}{\partial \delta_{3}}, q_{\delta 3}=\frac{\partial y}{\partial \delta_{3}}, p_{\delta 4}=\frac{\partial x}{\partial \delta_{4}}$ and $q_{\delta 4}=\frac{\partial y}{\partial \delta_{4}}$. We can see that state variables $x_{1}$ and $x_{2}$ serve as the inputs in equations (12) and (13), and, therefore, the sensitivity of the system to the uncertainties is a function of the trajectories of $x_{1}$ and $x_{2}$. Since the system in equation (1) is observable, $x_{1}$ and $x_{2}$ or their estimates are computable at each operating point and we have no problems in computing the sensitivity of the system to the uncertainties. Sensitivity computations to $f$ is computed as:

$$
\begin{array}{ll}
\dot{p}_{f_{1}}=-p_{f_{1}}+1 & \dot{p}_{f_{2}}=p_{f_{1}}-2 p_{f_{2}} \\
q_{f_{1}}=p_{f_{1}} & q_{f_{2}}=p_{f_{2}} .
\end{array}
$$

Since the fault is additive the states do not appear in equation (14), the sensitivity of residual $r_{1}$ to fault $f$ is not a function of the operating region.

Even though the sensitivity of states to faults and uncertainties are not part of the real system dynamics and we only need to compute them to analyze the performance of our residuals, it is important to prove the boundedness of these dynamic variables. Unboundedness of these variables increase computational expenses over time and eventually can stop the computation process and supervisory system operation. The stability of equations (10) and (11) is discussed in the following theorem.
Theorem 1: Given a stable linear system (1), sensitivity of states and outputs to uncertainties and faults represented by equations (10) and (11) are stable.

Proof: Consider equations (10) and (11). We can rewrite $\dot{p}_{\delta i}$ and $\dot{p}_{f}$ as:

$$
\begin{aligned}
\dot{p_{\delta i}} & =A p_{\delta i}+u_{i}, \\
\dot{p_{f}} & =A p_{f}+u_{f},
\end{aligned}
$$

where $u_{i}=\frac{\partial A}{\partial \delta_{i}} x+\frac{\partial B_{u}}{\partial \delta_{i}} u+\frac{\partial B_{f}}{\partial \delta_{i}}$ and $u_{f}=\frac{\partial A}{\partial f} x+\frac{\partial B}{\partial f} u+$ $\frac{\partial B_{f}}{\partial f}$. Since the linear system (1) is stable, $x$ is bounded and consequently $u_{i}$ and $u_{f}$ are bounded and equations (15) and (16) represent linear systems with stable system matrix $A$ and bounded inputs. Therefore, $p_{\delta i}$ and $p_{f}$ are stable. Considering stability of $x, q_{\delta i}$ and $q_{f}$ are stable too.

2) Residual sensitivity to uncertainties and fault: Using equations (10) and (11) we can derive sensitivity of residual $r(y, u)$ to fault $f$ and uncertainty $\delta_{i}$ as:

$$
\begin{aligned}
\frac{\partial r}{\partial \delta_{i}} & =\frac{\partial r}{\partial y} \frac{\partial y}{\partial \delta i}=\frac{\partial r}{\partial y} q_{\delta i}, \\
\frac{\partial r}{\partial f} & =\frac{\partial r}{\partial y} \frac{\partial y}{\partial f}=\frac{\partial r}{\partial y} q_{f} .
\end{aligned}
$$

Note that it is assumed that $\frac{\partial u}{\partial f}=0$ and $\frac{\partial u}{\partial \delta i}=0$. Consider the running example (3) and equations (12), (13) and (14). We compute $\frac{\partial r_{1}}{\partial \delta_{1}}, \frac{\partial r_{1}}{\partial \delta_{2}}$ and $\frac{\partial r_{1}}{\partial f}$ as:

$$
\frac{\partial r_{1}}{\partial \delta_{1}}=-x_{1} \quad \frac{\partial r_{1}}{\partial \delta_{2}}=0 \quad \frac{\partial r_{1}}{\partial \delta_{3}}=u_{1} \quad \frac{\partial r_{1}}{\partial \delta_{4}}=0 \quad \frac{\partial r_{1}}{\partial f}=1 .
$$

$r_{1}$ is not sensitive to $\delta_{2}$ and $\delta_{4}$, its sensitivity to $\delta_{1}$ is a function of the state variable $x_{1}$ and its sensitivity to $\delta_{3}$ is a function of the input $u_{1}$. Also independent of the operating point, its sensitivity to fault $f$ is always 1 . Considering | $\delta_{i} \mid \leq \Delta_{i}$ for $i=1,2,3,4$ and $F_{m}$ as the minimum absolute value of $f$ that we wish to detect, the detectability ratio of $f$ by $r_{1}$ is:

$$
D\left(f \mid r_{1}\right)=\frac{F_{m}}{F_{m}+\Delta_{1}\left|x_{1}\right|+\Delta_{3}\left|u_{1}\right|} .
$$

Considering $r_{2}$ we have:

$$
\begin{array}{lll}
\frac{\partial r_{2}}{\partial \delta_{1}}=-x_{1} & \frac{\partial r_{2}}{\partial \delta_{2}}=-2 x_{1}+2 x_{2}-2 u_{2} & \\
\frac{\partial r_{2}}{\partial \delta_{3}}=0 & \frac{\partial r_{2}}{\partial \delta_{4}}=u_{1}+u_{2}+\dot{u}_{2} & \frac{\partial r_{2}}{\partial f}=1 .
\end{array}
$$

We can see that both of the residuals have the same sensitivity to fault $f$ but their sensitivity to uncertainties are different. The detectability ratio of $f$ by $r_{2}$ is given by equation (22).

Fig. 3 represents two trajectories of the system corresponding to $u_{1}$ and $u_{2}$ for the running example (3). In this case fault $f=1$ occurs at time $t=120 \mathrm{~s}, \delta_{1}=-0.095$, $\delta_{2}=0.095, \delta_{3}=0.095$ and $\delta_{4}=0.04$. Given $\Delta_{1}=\Delta_{2}=$ $\Delta_{3}=0.1, \Delta_{4}=0.05$ and $F_{m}=0.5$, the absolute value of residuals $r_{1}$ and $r_{2}$ and the corresponding detectability ratios are presented in Fig. 4. The fault occurs at $t=120 \mathrm{~s}$, therefore, the residuals should both be zero for $t<120$ in the 


$$
D\left(f \mid r_{2}\right)=\frac{F_{m}}{F_{m}+\Delta_{1}\left|x_{1}\right|+\Delta_{2}\left|-2 x_{1}+2 x_{2}-2 u_{2}\right|+\Delta_{4}\left|u_{1}+u_{2}+\dot{u}_{2}\right|} .
$$

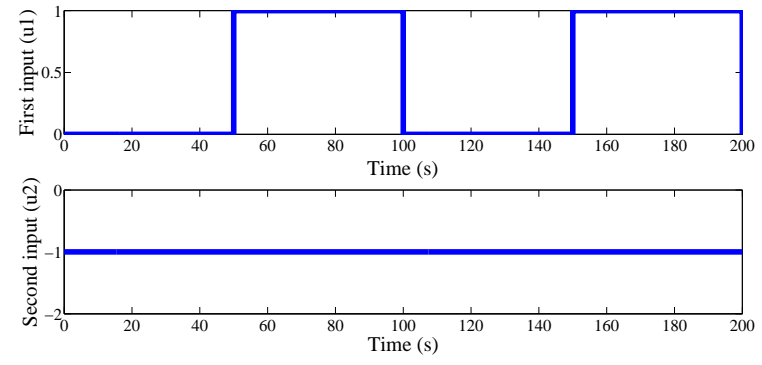

Fig. 3. Inputs of the system.

ideal case. But because of uncertainties the value of residuals varies according to the state and input signal variations. An interesting observation is that the detectability ratio for each residual in this time interval is maximum when the residual is close to zero, and decreases as the residual deviates from zero. So we can say a residual with higher detectability ratio is less likely to produce false alarms. After $t=120 \mathrm{~s}$, when there is a fault in the system, the residual with higher amplitude ( $r_{1}$ in this example) has a higher detectability ratio as well. So we can say the residual with higher detectability ratio is more likely to report the fault and also because of higher sensitivity it can detect smaller fault magnitudes.

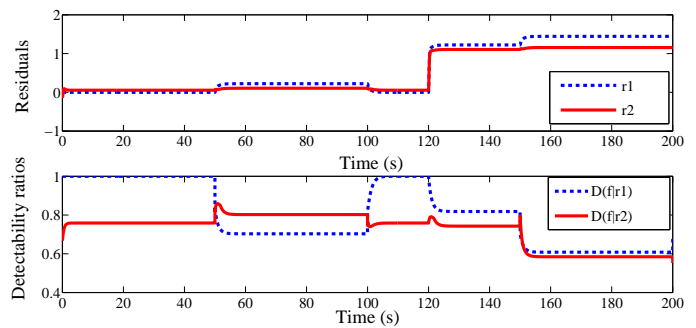

Fig. 4. Residuals and their corresponding detectability ratios.

The main idea of this paper is to track the detectability ratio online, and choose the best residual for each of the operating regions of the system. If we use the following hybrid residual for the system we will have a residual with maximum robustness to the uncertainties and sensitivity to the fault,

$$
r= \begin{cases}r_{1} & \text { if } D\left(f \mid r_{1}\right) \geq D\left(f \mid r_{2}\right) \\ r_{2} & \text { otherwise. }\end{cases}
$$

Fig. 5 represents $r_{1}, r_{2}$ and $r$. As we can see $r$ obviously has a better performance rather than $r_{1}$ and $r_{2}$.

\section{Case Study}

In order to show how detectability ratio can be applied to choose the best residual in real systems with uncertainties, we analyze a two tank system shown in Fig. 6 .

The system consists of an input flow source $(S)$ with volumetric flow rate $u$, two tanks $\left(T_{1}\right.$ and $\left.T_{2}\right)$ and two

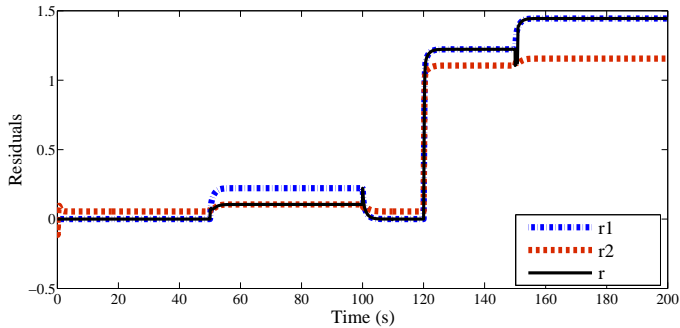

Fig. 5. Two residuals and their combination.

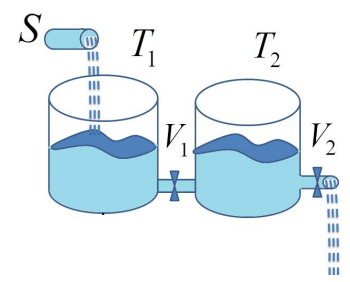

Fig. 6. Two tanks system configuration.

valves ( $V_{1}$ and $V_{2}$ ). Two sensors measure the pressure of the first and the second tank ( $p_{1}$ and $\left.p_{2}\right)$. The first tank's nominal capacity is $C_{1}$ and the second one's is $C_{2}$. Valves $T_{1}$ and $T_{2}$ have nominal resistances of $R_{1}$ and $R_{2}$, respectively. Assume that there is $\delta_{1}$ uncertainty in $C_{1}, \delta_{2}$ uncertainty in $C_{2}$, a possible fault $f$ in $R_{1}$ and $\delta_{3}$ is the uncertainty in $R_{2}$. Considering $p_{1}$ and $p_{2}$ as the system state variables the system state space model is shown in equation (24), $y_{1}$ and $y_{2}$ represent the measurements. Equation (25) represents a simple proportional controller to regulate the pressure in the first tank.

$$
u=K\left(r(t)-y_{1}\right),
$$

where $K$ is the controller gain and $r(t)$ is the desire trajectory of the pressure in $C_{1}$. To detect fault $f$ two residuals $r_{1}$ and $r_{2}$ are designed.

$$
\begin{array}{r}
r_{1}: \dot{y_{1}}-\frac{K\left(r(t)-y_{1}\right)}{C_{1}}+\frac{y_{1}-y_{2}}{R_{1}}, \\
r_{2}: \dot{y_{2}}-\frac{y_{1}-y_{2}}{R_{1} C_{2}}+\frac{y_{2}}{R_{2} C_{2}} .
\end{array}
$$

$R_{1}$ appears in both of the residuals and if there is no uncertainty in the model either $r_{1}$ or $r_{2}$ can be used to detect $f$. In the presence of uncertainty each residual is affected differently, and the sensitivity is also a function of the operating region of the system. In order to evaluate the performance of each residual as a function of the operating region, we compute the detectability ratio in each region. Step function $u(t)$ is considered as the desired trajectory for the first tank pressure. Three uncertainties $\delta_{1}=-.09$ , $\delta_{2}=0.08$ and $\delta_{3}=0.145$, are considered. Also it is assumed that we know, $\Delta_{1}=\Delta_{2}=0.1, \Delta_{3}=0.2$ and $F_{m}=1$. Fault $f=1$ is considered as a fault in $R_{1}$ and it 


$$
\begin{array}{r}
\left(\begin{array}{c}
\dot{p_{1}} \\
\dot{p_{2}}
\end{array}\right)=\left(\begin{array}{cc}
\frac{-K}{C_{1}\left(1+\delta_{1}\right)}+\frac{-1}{R_{1}(1+f) C_{1}\left(1+\delta_{1}\right)} & \frac{1}{R_{1}(1+f) C_{1}\left(1+\delta_{1}\right)}-1 \\
R_{1}(1+f) C_{2}\left(1+\delta_{2}\right) & \frac{-1}{R_{2}\left(1+\delta_{3}\right) C_{2}\left(1+\delta_{2}\right)}
\end{array}\right)\left(\begin{array}{l}
p_{1} \\
p_{2}
\end{array}\right)+\left(\begin{array}{c}
\frac{K}{C_{1}\left(1+\delta_{1}\right)} \\
0
\end{array}\right) r(t), \\
\left(\begin{array}{l}
y_{1} \\
y_{2}
\end{array}\right)=\left(\begin{array}{ll}
1 & 0 \\
0 & 1
\end{array}\right)\left(\begin{array}{l}
p_{1} \\
p_{2}
\end{array}\right) .
\end{array}
$$
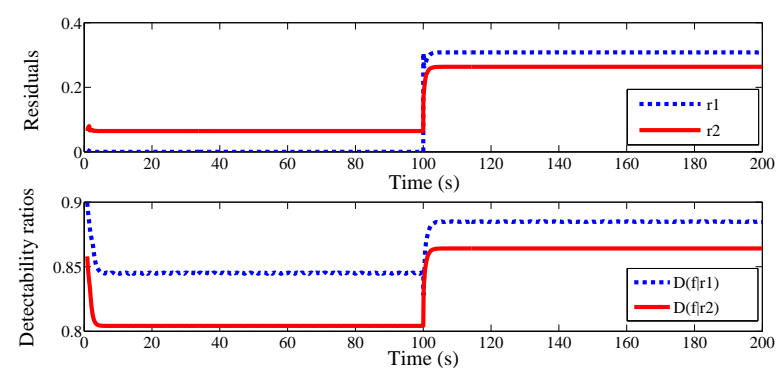

Fig. 7. Residuals and the corresponding detectability ratios for two tank system.

occurs at $t=100 \mathrm{~s}$. The absolute value of the residuals $r_{1}$ and $r_{2}$ and the detectability ratio of each is computed and plotted in Fig. 7. We can see from this figure that residual $r_{1}$ has a lower amplitude than $r_{2}$ before fault occurs and higher value after the fault happens. So this residual shows better performance to detect $f$ and is less likely to cause false alarms when there is no fault in the system. We can see this in the detectability ratio of this residual, which is always higher than detectability ratio of $r_{2}$. The better performance of $r_{1}$ over $r_{2}$ is not surprising at all. Analyzing $r_{1}$, we see that the only source of uncertainty in this residual is uncertainty in $C_{1}$, and $C_{1}$ is multiplied by $\left(r(t)-p_{1}\right)$ in the numerator, which is the tracking error and is expected to be a small quantity. But on the other hand in $r_{2}, R_{2}$ and $C_{2}$ are two sources of uncertainty and the terms in the numerators are not expected to be a small quantity in this trajectory.

In the cases that the trajectory is known, we can do all the analysis offline and find the best residual at design time. Offline residual selection [13] can save computational expenses associated with online residual selection. Because we do not need to compute the sensitivity of state and residuals to the fault and uncertainties at each operation point. It is important especially when the systems has many state variables and parameters with different sources of uncertainty. Moreover, in the offline method the system does not need to be observable because we run the analysis only for the given trajectories, we do not need to measure or estimate the state variables. For systems with fast dynamic we believe that it is best to set a threshold in the switching process to avoid chattering between the residuals.

\section{CONCLUSIONS}

The detectability ratio is defined as a quantitative measure of the residual detectability performance. It provides a measure to find the residual which is most sensitive to the fault and robust to the uncertainties at different regions of system operation. Two different examples are presented to evaluate and demonstrate the effectiveness of this approach. For online residual selection the detectability ratio is used to select the best residual for different operating points. In general, it is possible to achieve better detection by using more than one residual. For future work, we will propose a framework to consider all the potential residuals and design an optimal residual with the highest sensitivity taking into account that different faults may occur, and robustness to the uncertainties may change in different operating regions of system behavior. We can also extend the detectability analysis to isolability analysis that involves a number of potential single faults that can occur in the system.

\section{REFERENCES}

[1] C. Chen and R. Patton, Robust Model-Based Fault Diagnosis For Dynamic Systems, ser. Kluwer International Series on Asian Studies in Computer and Information Science, 3. Kluwer, 1999.

[2] W. Manners, Classification and Analysis of Uncertainty in Structural Systems. Springer Berlin Heidelberg, 1991.

[3] K. B. Laskey, "Model uncertainty: theory and practical implications," IEEE Transactions on Systems, Man and Cybernetics - Part A. Systems and Humans, vol. 26, 1996.

[4] R. S. Mangoubi, Robust estimation and failure detection: A concise treatment. Springer Publishing Company, Incorporated,, 2012.

[5] A. Marcos, S. Ganguli, and G. Balas, "An application of $\mathrm{H}_{\infty}$ fault detection and isolation to a transport aircraft," Control Engineering Practice, vol. 13, no. 1, pp. 105-119, 2005.

[6] C. Join, J. C. Ponsart, D. Sauter, and D. Theilliol, "Nonlinear filter design for fault diagnosis: application to the three-tank system," IEE Proceedings-Control Theory and Applications, vol. 152, no. 1, pp. 55-64, 2005.

[7] M. A. Djeziri, R. Merzouki, B. O. Bouamama, and G. DauphinTanguy, "Robust fault diagnosis by using bond graph approach," Mechatronics, IEEE/ASME Transactions on, vol. 12, no. 6, pp. 599611, 2007.

[8] V. Puig, J. Quevedo, T. Escobet, F. Nejjari, and S. De Las Heras, "Passive robust fault detection of dynamic processes using interval models," Control Systems Technology, IEEE Transactions, vol. 16, no. 5, pp. 1083-1089, 2008.

[9] I. Hwang, S. Kim, Y. Kim, and C. E. Seah, "A survey of fault detection, isolation, and reconfiguration methods," IEEE Transactions on Control Systems Technology, vol. 18, no. 3, pp. 636-653, 2010.

[10] D. Eriksson, E. Frisk, and M. Krysander, "A method for quantitative fault diagnosability analysis of stochastic linear descriptor models," Automatica, 2013.

[11] _ - "A sequential test selection algorithm for fault isolation," in 10th European Workshop on Advanced Control and Diagnosis, Copenhagen, Denmark, 2012.

[12] L. R. Petzold and U. M. Ascher, Computer methods for ordinary differential equations and differential-algebraic equations. Siam, 1998, vol. 61.

[13] H. Khorasgani, D. Eriksson, G. Biswas, E. Frisk, and M. Krysander "Off-line robust residual selection using sensitivity analysis," in 25th International Workshop on Principles of Diagnosis (DX-14), Graz, Austria, Accepted for publication.

[14] R. Serban and A. C. Hindmarsh, "CVODES, the sensitivity-enabled ODE solver in SUNDIALS," in Proceedings of the 5th International Conference on Multibody Systems, Nonlinear Dynamics and Control, Long Beach, CA, 2005.

[15] D. Henry and A. Zolghadri, "Design and analysis of robust residual generators for systems under feedback control," Automatica, vol. 41, no. 2, pp. $251-264,2005$. 